\title{
Commentary: Forward to the Special Issue on Cough in Current Otorhinolaryngology Reports
}

\author{
Kenneth W. Altman ${ }^{1}$ \\ Published online: 14 May 2019 \\ (C) Springer Science+Business Media, LLC, part of Springer Nature 2019
}

Chronic cough has gained substantial notoriety over the last few years, with growing recognition of its prevalence and an increasing awareness of the CHEST Cough Guidelines. One of the interesting aspects of the cough is that it is not a disease in and of itself, but rather an indication of a disease that's triggering the cough as a protective or adaptive reflex. Similarly, since the sound is centered in the larynx, cough as a manifestation of disease is susceptible to multiple stimuli from the aerodigestive tract. These multifactorial contributions create a synergy, priming receptors, and central neurologic reflexes to be more excitable with additional stimuli. The Cough Guidelines are comprehensive in their review of the scientific literature related to over 40 clinical topic areas, with more than 50 interprofessional experts from 11 disciplines globally, vetting a consensus to create expert recommendations for clinical care. However, there are important subjects related to real life management of cough that need to be communicated, which are outside the confines of a condensed recommendation.

This issue on Cough in Current Otolaryngology Reports expands on the existing literature with clinically relevant reviews that are important to clinical practice.

- "Occupational and environmental exposures and their role in chronic cough," by Dr. Tarlo, heightens our awareness of the many caustic occupational and environmental stimuli that can cause a cough through direct stimulation of cough receptors, as well as causing respiratory tract disease that further triggers a cough. Although it's an area that requires more study, these exposures should be discussed in the routine evaluation of all patients with cough.

This article is part of the Topical Collection on Chronic Cough

Kenneth W. Altman

Kenneth.altman@bcm.edu

1 Baylor College of Medicine, Houston, TX, USA
- "The spectrum of non-asthmatic airway diseases contributing to cough in the adult," by Dr. Braman, expands on the routine understanding of pulmonary disease by otolaryngologists which generally includes asthma, bronchitis, and tumors. He discusses an important spectrum of nonasthmatic pulmonary diseases that should be on the differential of all clinicians treating cough.

- "Therapeutic targets for the treatment of chronic cough," by Dr. McGarvey and colleagues, provides a concise yet comprehensive summary of the targets for cough suppression therapy, noting some emerging medications that hold promise for patients with severe cough or those that are refractory to treatment.

- "The larynx as a target for treatment in chronic refractory cough," by Dr. Vertigan, demonstrates the important contribution of laryngeal desensitization and cough suppression therapies. Here, behavioral modification to maladaptive protective mechanisms and retraining ingrained reflexes are also valuable in cough suppression therapy.

- "Modern challenges managing chronic cough in otolaryngology" by Drs. Altman and Irwin addresses the outlook in creating a cohesive approach to caring for cough patients, both in the clinic and globally.

These are some of the many interesting and relevant topics that elevate our scientific understanding and enhance clinical care in cough patients. Expanding the discussion to include practicing otolaryngologists to help serve unmet need in complex cough patients elevates quality of life and the public health.

Publisher's Note Springer Nature remains neutral with regard to jurisdictional claims in published maps and institutional affiliations. 